\title{
LOS IDEALES DE LA CABALLERÍA EN LA SOCIEDAD MEDIEVAL
}

MaRía PURIFICACIÓN ViLa

Universidad Politécnica de Madrid

En el siglo XIV, la corte real no era simplemente una institución social, sino que mediante ella era gobernado el país y muchos de los cortesanos que a ella pertenecían se dedicaban a los asuntos de estado. La corte se trasladaba de un lado a otro, probablemente en busca de mejores servicios y alimentos. De esta forma, el Gobierno operaba en diferentes partes del país, pero más adelante se asentó permanentemente en Westminter.

Las relaciones de Chaucer con la corte le permitieron conocer a esta familia de clase noble que nos relata en The Canterbury Tales, representada por el Caballero, su hijo el Escudero y su criado el Arquero.

En «The Knight's Tale», Chaucer destaca los peligros que se derivan del poder omnipotente de reyes y monarcas. En este cuento, el Duque Teseo ordena que Palamón y Arcite que habían sido hechos prisioneros en la guerra que había sostenido Atenas con Tebas, fueran encerrados en una torre hasta el resto de sus días.

Los poemas de Chaucer indican, por una parte, los gustos del público para quien han sido descritos, reflejando la sociedad medieval, los valores y actitudes propias de la época.

La caballería tuvo un importante papel en la vida medieval. En The Canterbury Tales, Chaucer nos presenta la figura de un caballero del que hace una 
descripción idealizada, destacando su lealtad, honor y cortesía, valores esenciales para todo aquel que pretendiese aspirar a ser armado caballero.

El caballero medieval tenía una profesión perfectamente compatible con su religión, y los ideales de la caballería estaban muy relacionados con los de la cristiandad. La misma ceremonia en la que era nombrado caballero presentaba grandes semejanzas con los ritos religiosos. Los ideales de la caballería se apoyaban en los sentimientos de bravura y lealtad, siendo uno de los principales objetivos del caballero obtener honores, como podemos observar en "The Franklin's Tale», donde el marido de Dorigena, Averargo, deja a su bella esposa para ir a la corte del rey Arturo, a fin de obtener glorias y honores.

La sociedad medieval aparece normalmente dividida en tres grupos o estamentos, de los cuales los caballeros ocupan el primer lugar, seguido por la Iglesia y los laicos. El tercer nivel está formado por los burgueses y los siervos.

The Knight, es normalmente considerado como una figura idealizada de la caballería. Según J. A. Manly (1929-1959), el Caballero era «a figure at once realistic and typical of the noble and adventurous idealists of his day". También M. Bowden (1969), afirma que este caballero personificaba los ideales de la caballería y era:

The champion of the Church, the righteous and implacable enemy of the infidel, the compassionate protector of the weak and opressed, the defender of all Rights and Justice.

En este mismo sentido, J. Mann (1973), opina que el caballero es un acérrimo defensor de la cristiandad y la quintaesencia de la caballería:

It seems clear that Chaucer wishes us to accept the Knight's motivation as religious. Not only do the historians tell us that all the campaigns named were against the heathen, but the vocabulary of the portrait stresses the opposition between

"cristendeom" and "heathenesse", that the Knight is "Cristen man», who has "foughted for oure feith" and campaigned against the "heathen".

Todas estas ideas se basan en la descripción que Chaucer hace del Caballero como «a worthy man» y desde el primer momento que montó a caballo «he loved chevalrie, trouthe and honour, fredom and courtesie» (T.C.T. I, 45-46). 
Las líneas siguientes sirven para introducir la serie de campañas y batallas en las cuales había participado y que son una demostración de la dedicación del caballero a la defensa de la cristiandad por medio de las armas:

Ful worthy was he in his lordes werre, And therto hadde he riden, no man ferre

As wel in cristendom as in hethenesse,

And evere honoured for his worthynesse.

(T.C.T. I 47-50)

Esta interpretación de la figura del caballero es criticada por otros autores, como $T$. Jones, que opina:

There is no mention of any family background, no coat-of-arms, no shield, no belt (crucial to the truly agentil» or noble knight), no manorial estates. He shows no interest in the courtly pastimes of hunting, hawking or courtly love.

His dress is shabby, his retenue small, and his life-long career on the battlefield has been exclusively abroad and has apparently mis. sed out on all the great English victories of the period - such as Crecy. Poitiers and Najera- on which the reputation of English chivalry in the fourteenth rested.

T. Jones (1982), demuestra en su obra que The Knight, en lugar de ser un verdadero representante de la caballería, posee muchas características comunes de los caballeros mercenarios. En el siglo XIV, estos caballeros fueron muy numerosos y guerrearon por toda Europa en las llamadas «Free-Companies», impulsados por el único afán del dinero y de una vida independiente. De esta manera, los ejércitos, en vez de estar formados por los habitantes de una nación, tenían un gran número de caballeros mercenarios. Esta nueva situación, propició el que se formaran estas compañías guerreras en diferentes países y durante largos períodos de tiempo, como sucedió con la guerra de los Cien años entre Francia e Inglaterra.

Los caballeros mercenarios supusieron también un peligro al encontrarse muchas veces sin empleo y ser la guerra su único oficio conocido, por lo que en muchas zonas, sobre todo de Francia e Italia, se dedicaron exclusivamente a la rapiña y los daños causados sólo fueron superados por la Peste Negra. 
En 1360, el Tratado de Bretigny produjo el cese temporal de las hostilidades entre Inglaterra y Francia. Aun cuando esta paz fue bien recibida por ambas partes, muchos de los soldados que habían hecho la guerra durante los últimos veinte años, se encontraron desocupados y se agruparon en compañías bajo el mando de capitanes, tales como John Hawkwood. Este valiente caballero, había realizado grandes hechos de armas, y según refiere Froissart en sus Crónicas (1988), pensó que al ser un pobre y joven caballero, no le sería de ningún provecho regresar a Inglaterra, por lo que se trasladó a Italia, donde ofreció sus servicios al Papa Urbano y a otros personajes destacados de la época.

Estos caballeros obtuvieron gran fama y dinero por sus conquistas y era frecuente conseguir como recompensa la mano de la hija de uno de los señores a los que servía. Así, el Gran Duque Visconti de Milán, casó a una de sus hijas con John Hawkwood, convirtiéndole así en un noble y rico propietario.

Cuando Chaucer visitó Milán, en 1368, fue envidado por el rey para discutir «certain affairs touching the expedition of the King's war». La guerra contra Francia se había reanudado de nuevo y por este motivo se enviaba una embajada a Lombardía para pedir ayuda militar y financiera.

No resulta, por tanto, extraño que Chaucer quisiera servirse de la figura del Caballero para satirizar a esos soldados mercenarios que habían encontrado fama y fortuna en otras tierras, fuera de las posesiones de la corona inglesa. Parece cierto que los viajes a tierras extrañas tenían una gran fascinación para el pueblo, pero los caballeros que abandonaban su propio país para correr aventuras en tierras lejanas, fueron casi siempre objeto de crítica por sus contemporáneos.

Inglaterra había quedado diezmada por la Peste Negra y el número de caballeros era realmente escaso y su verdadero trabajo consistía en ocuparse de defender su país del acoso de sus enemigos, al mismo tiempo que defendían su familia y su patrimonio.

Con esta idea, Chaucer critica irónicamente el comportamiento del caballero quien «Ful worthy was he in his lordes werre/And therto hadde he riden, no man ferre...» (T.C.T. I 47-48). Al ponerse al servicio de «his lordes werre» y al dedicar a esta causa más tiempo que cualquier otro hombre, parece lógico pensar que el caballero no había servido al rey de Inglaterra en remotos lugares, tales como España, Turquía, Africa, Lituania y Rusia, cuando las luchas del rey inglés se producían en su propio país, así como en sus posesiones de Francia y Escocia.

En la época en que Chaucer describía al Knight en «The General Prologue», las cruzadas habían perdido la importancia y fervor de otros tiempos y muchos se preguntaban si el dedicarse a matar infieles era el mejor siste- 
ma para convertirlos a la religión cristiana. Aun cuando algunos pensaban que la Iglesia poseía ciertos derechos para conquistar los Santos Lugares, no existía ninguna ley que permitiese la guerra contra otros países, tales como Francia, Lituania o Turquía, donde según Chaucer, el Caballero había luchado durante muchos años. Era cada vez más frecuente la idea de que los «hethens» tenían unos sentimientos tan humanos como los cristianos.

Una razón pragmática por la que había disminuido el entusiasmo por las cruzadas a la «hethenesse», era la idea que tales conquistas resultaban inútiles. Esto se cumplía, especialmente en relación con los lugares donde el Caballero había combatido. Alejandría había sido ganada y perdida por Pedro de Chipre en un corto espacio de tiempo, tras producirse una tremenda masacre. El único resultado que se obtuvo aparte de los daños ocasionados por la matanza anterior, fue la de interrumpir los intercambios comerciales con Oriente.

El Caballero, no solamente había participado en la toma de Alejandría, sino que se había sentado en la mesa de los caballeros de la Orden Teutónica, luchando en «las Cruzadas» de Polonia y Prusia. También había combatido en Lituania, donde él había «reysed» más a menudo que cualquier otro cristiano de su rango, pero según se nos refiere tuvo escaso éxito, ya que se capturaron o mataron ochocientos infieles, de los que tan sólo ocho se convirtieron al cristianismo.

At Alisaundre he was whan it was wonne.

Ful ofie tyme he hadde the bord bigonne

Aboven alle nacions in Pruce;

In Lettov hadde he reysed and in Ruce.

No Cristen man so ofte of his degree.

(T.C.T. I, 51-55)

La actitud de la sociedad ante la importancia de los mercenarios están reflejados en la literatura de la época que no podía permanecer pasiva ante estos caballeros que ofrecían con su vida aventurera temas sobrados para escribir libros que enaltecieran sus virtudes y los utilizaran como ejemplo. El empleo de estos caballeros en las empresas guerreras, resultaba muy claro en una época en la que el suelo era escaso e incierto, por lo que el pillaje era a menudo el único medio de subsistencia para aquellos caballeros que abandonaban a su familia y amigos para luchar en tierras lejanas. Salvar las almas de los infieles o seguir el espíritu de aventura, no era el verdadero motivo que impulsó a estos caballeros, según afirman muchos escritores de la época. Gower dice que la única razón por la que 
estos guerreros viajaban «over the grete Se» o a Prusia, Rodas o Tartaria, era la de ganar «worshipe», es decir, la fama y el honor que sirviera para impresionar y obtener el amor de su dama. Utilizaban como táctica la de ir precedidos de heraldos que comunicaban su llegada por lo que recibían a cambio dinero y vestidos que eran motivo de comentario y servían para aumentar su fama.

El poeta francés Guillaume de Machaut, opinaba igualmente que el hecho de ir a otras tierras a combatir contra los infieles era tan sólo una manera de impresionar a las damas. Incluso el cronista Froissart que tanto ensalza a la caballería, señala que tanto Francia como otros lugares servían para que los «men of arms» aumentaran su fortuna y celebridad. Al marcharse a combatir a tierras lejanas, no demostraban un gran aprecio por su familia, ni por cumplir con sus obligaciones, encontrándose muchas veces a su vuelta con que su hogar y patrimonio había sido destruido por alguna revuelta. La sustitución del caballero feudal por el soldado de fortuna, supuso también un cambio en el orden social. Las relaciones familiares patriarcales y feudales, se basaban entonces en unas bases puramente económicas que erosionaban los valores sociales de la época y restaban en gran parte importancia a la lealtad y el honor. No resulta, por tanto, sorprendente el que Chaucer haya elegido la figura de este caballero para satirizar a la sociedad de su tiempo.

El propio Chaucer identifica en uno de sus anteriores poemas los sitios donde el Knight había luchado, como los típicos lugares a los que las damas enviaban a sus enamorados caballeros como prueba de amor.

En The Book of the Duchess. The Black Knight, se enorgullece de que su dama Blanche no siga esta costumbre con sus pretendientes:

Ne sende men into Walakye,

To Pruyse and into Tartarye,

To Alysaundre, ne into Turkye,

And byd him faste, anoon that he

Go hoodles to the Drye Se,

And come hom by the Carrenar;

And seye, «Sir, be now ryght war

That I may of yow here seyn

Worshyp, or that ye come ageyn!

She ne used no suche knakkes smale."

(T.B.D., 1024-1033)

Las cruzadas a tierras de infieles eran consideradas por muchos contemporáneos, no como una misión evangélica, sino como una excusa para llenar 
su ambición y vanidad personal. Si existía una fuerte crítica a las cruzadas en «hethenesse», mayor debía ser la oposición contra las cruzadas dentro de la cristiandad, pues la lucha no era contra los infieles sino contra los propios cristianos. En el siglo XIV era frecuente que el Papa sostuviera un gran número de luchas contra sus oponentes dentro de la propia Iglesia, hasta el punto de mostrar el escandaloso espectáculo de entrar en batalla los partidarios de diferentes papas que trataban de dignificar estos hechos dándoles el título de cruzada.

Lo que realmente indignaba en aquella época, era el que la Iglesia fuese uno de los principales estados que utilizaban a las «Free Companies» para llevar a cabo estas guerras. Como explica Froissart, el caballero John Hawkwood, luchó a sueldo durante varios años, junto con sus ejército de soldados mercenarios en las guerras de Roma, a favor del Papa Urbano VI, contra las demás ciudades italianas y el antipapa Clemente de Avignon. La mayoría de estos mercenarios y el propio Hawkwood, eran ingleses, razón por la cual estos hechos eran muy conocidos en Inglaterra. No es de extrañar que Chaucer destacase al describir la figura del caballero que él había combatido tanto «in cristendom» as «in hethenesse» (T.C.T. I, 49).

J. Mann (1973), al examinar el retrato del Caballero, opina que su figura carece de una verdadera estructura social, ya que no indica claramente la finalidad de sus actividades guerreras, que aun cuando tenían un carácter religioso, su verdadero motivo no era el de salvar las almas de los infieles.

El Caballero de Chaucer es un «verray, parfit gentil Knight» (T.C.T. I, 72). Al igual que el resto de los peregrinos es un profesional que no demuestra de forma palpable a lo largo de toda su descripción, la importancia que tiene su profesión al defender la vida y hacienda de los demás personajes. Seguramente, porque Chaucer satiriza el carácter mercenario de estos caballeros guerreros.

Acompañaba al Caballero «his sone, a yong Squier» (T.C.T. I, 79). Esta relación familiar que se establece entre ambos, parece derivar por otro lado de la conexión que existía en aquella época entre ambas profesiones. Era frecuente que los caballeros enviasen a sus hijos a servir a las órdenes de otro señor para aprender su oficio.

Según cuenta el libro Lordre de Chevalerie la ciencia y la enseñanza del orden de la caballería era la de que el Caballero enseñara a su hijo a cabalgar en su juventud. Era conveniente que el hijo del caballero, mientras sirviese como escudero supiera cuidar el caballero, al igual que tenía que tener en cuenta que debía ser siervo antes que señor. Por ese motivo, todo caballero debía poner a su hijo al servicio de otro caballero, a fin de aprender a trinchar la carne, 
a servir, vestir y colocar la armadura. El Escudero de Chaucer se adapta a este modelo:

Curteis he was, lowely, and servysable, And carf biforn his fader at the table.

El Escudero, al igual que su padre el Caballero, es «Curteis» y también generoso y servicial con los demás. Atiende con amabilidad a todos los caballeros a los que sirve, especialmente a su padre, por lo que «carf biforn his fader at the table». Esta deferencia tiene un doble motivo: la de ser una muestra de respeto filial, así como una demostración de servicio a un caballero. Su cortesía tiene distintos fundamentos de los de su antecesor, ya que el Caballero era «as seeke as is a mayde/He nevere yet no vileynye no sayde» (T.C.T. I, 69-70). $\mathrm{El}$ joven escudero es descrito por Chaucer como «a lovyere and a lusty bacheler/With lokkes crulle as they were leyd in presse» (T.C.T. I, 80-81). Al describir al Caballero, Chaucer emplea un lenguaje lleno de palabras selectas que indican su condición de noble, mientras que cuando se trata de su hijo el Escudero, emplea palabras más corrientes que nos muestran su carácter juvenil e ingenuo.

Según M, Bowden (1969), el Escudero recuerda al propio Chaucer en su juventud, al describir de forma retrospectiva sus costumbres y éxito con las damas. Su condición de «bacheler» se debe al estar próximo su nombramiento de caballero, de acuerdo con las normas medievales. Este término también se usa al tratarse de un joven caballero, como hace Chaucer en "The Wife of Bath's Tale», al hablarnos de su protagonista. Este joven caballero se encontraba en la Corte del Rey Arturo, como nos refiere el poeta, al decir que este rey «hadde in his hous a lusty bacheler» (T.C.T. III, 883).

El Escudero, además de tener los cabellos rizados («lokkes crulle»), tenía un aspecto vigoroso («greet strengthe»), y también proporcionado («evene lengthe»), al mismo tiempo que poseía una gran agilidad («delyvere»). Por sus características físicas, podía ser considerado tanto «dygne» como «worthy» para poder ser admitido en la «ordre of Chyvalrie», a pesar de su juventud.

Of twenty yeer of age he was, I gesse,

Of his stature he was of evene lengthe,

And wonderly delyvere, and of greet strengthe.

(T.C.T., I, 82-84). 
Por otra parte lordre de Chevalerie, critica el aspecto excesivamente cuidado y elegante que tenían algunos escuderos, por lo que son objeto de sátira y burla por parte de algunos escritores de la época. Chaucer, describe al joven Escudero que llevaba los clásicos vestidos y adornos que ridiculizan su figura:

With lokkes crulle as they were leyd in presse...

Embrouded was he, as it were a meede

Al ful of fresshe floures, whyte and reede,...

Short was his gowne, with sleves longe and wyde.

(T.C.T. I, 81-90; 93).

Según J. Mann (1973) era costumbre criticar el aspecto elegante de los caballeros mundanos que peinaban sus cabellos con extremada coquetería y que utilizaban métodos y adornos similares a los de las mujeres. Ocultaban sus delicadas manos bajo amplias mangas y llevaban cortos vestidos que permitían adivinar sus miembros. Esta apariencia afeminada y elegante, más que un signo de vanidad, nos parece una característica de ser «a lovyere» y de tratar de agradar a las mujeres.

El Escudero de Chaucer, parece contar con la aprobación indulgente del poeta y de los propios lectores que simpatizan con este personaje por su juventud y encantos personales. El Escudero, acompaña su canto al son de la flauta, y su música parece estar en armonía con sus modales y su forma de vestir:

Syngynge he was, or floytynge, al the day;

He was as fresh as is the month of May...

Wel koude he sitte on hors and faire ryde.

He koude songes make and wel endite,

Juste and eek dauce, and well purtreye and write.

(T.C.T. I, 91-92; 94-96)

La habilidad para cantar, danzar y componer la letra y música de sus canciones de amor era frecuente entre las damas y caballeros de la época. Chaucer en «The Franklin's Tale» describe un personaje llamado Aurelio que presenta rasgos semejantes a los del Escudero. Era igualmente un joven escudero que se 
adornaba y engalanaba como el mes de mayo y cantaba, danzaba y componía bellas canciones mejor que cualquier otro hombre.

"Upon this daunce, amonges othere men,

Daunced a squier biforn Dorigen,

That fressher was and jolyer of array.

As to my doom, than is the month of May.

He syngeth, daunceth, passynge any man/...

Of swich matere made he manye layes,

Songes, compleintes, roundels, virelayes.

(T.C.T. V, 925-929; 947-948)

El ideal cortés del Escudero se dirige fundamentalmente hacia el galanteo con las mujeres y la imagen de su amor apasionado está equilibrada con la comparación romántica de su figura con la del ruiseñor. Su «hoote» amor sugiere una pasión sexual, mientras que el «nythingale» recuerda un amor romántico:

«So hoote he lovede that by nyghtertale

He sleep namoore than dooth a nyghtyngale».

(T.C.T. I, 97-98)

De esta manera, Chaucer hace que el Escudero nos resulte algo enigmático, tanto por su aspecto personal como por la ingenuidad de sus amores, que también quedan reflejados en sus expediciones militares que tienen como finalidad la esperanza de ganar el favor de una dama. Este deseo amoroso, se combina con el servicio mediante las armas a la causa de Dios al participar en las guerras religiosas como lo hiciera su padre, el Caballero:

«And he hadde been somtyme in chyvachie

In Flaundres, in Artoys, and Pycardie,

And born hym weel, as of so litel space,

In hope to stonden in his Lady grace."

(T.C.T., I, 85-88) 
La devoción que siente el Escudero, tanto para amar como para guerrear y obtener los favores de su dama, resulta apropiada para su juventud y «lustiness». Chaucer hace compaginar la participación del Caballero y el Escudero en las guerras religiosas con los deseos que ambos muestran respectivamente «a sovereyn pris» y «his lady grace».

Al participar el Caballero en estas luchas en países tan lejanos, no resulta extraño que su vida azarosa impidiese que sus relaciones familiares fueran estables y permite suponer que debió tener a su hijo, el Escudero, con alguna de las damas que amó en su juventud, continuando así su estirpe y profesión.

El Caballero, el Escudero y el Arquero, forman la única familia que, como representantes de la nobleza, se dirigen en peregrinación a Canterbury. Aun cuando el Arquero no estaba unido por ningún lazo familiar con sus señores, mantenía con ellos una estrecha relación por su oficio; compartía con ellos las penalidades de la guerra y les atendía en tiempos de paz.

Así, en los siglos XIV y XV, la caballería era junto con la religión el concepto ético que dominaba la mente y el corazón de los hombres. Se consideraba, por tanto, a la caballería como la cumbre de todo sistema social. 


\section{BIBLIOGRAFIA}

BoITANI, P.: «The genious to improve an invention: transformations of The Knight's Tale». Chaucer Traditions. Studies in honour Derek Brewer. R. Mores and B. Windheat (eds.). Cambridge: Cambridge University Press, 1990.

BROOKS, D. y FowLER, A.: «The meaning of Chaucer's Knight's Tale». Geoffrey Chaucer's The Knight's Tale. H. Bloom (ed.), New York: Chelsea House Publishers, 1988.

BowDEn, M.: A comentary on the General Prologue to the Canterbury Tales. 2nd. ed. London, 1969.

COOPER, H.: «An opening: The Knight's Tale». Geoffrey Chaucer's The Knight's Tale. H. Bloom (ed.) New York: Chelsea House Publishers, 1988.

FroIsSART, J.: Crónicas. Madrid: Ediciones Siruela, 1988.

GORLACH, M.: «The Knight's Army», $N \& Q$. Vol. 20. 1973. pp. 363-365.

HulzingA, J.: The Waning of the Middle Ages. London: Penguin Books, 1924-1987.

JONES, T.: Chaucer's Knight. London: Eyre-Methuen, 1982.

KAHRL, S. J.: «Chaucer's Squire's Tale and the decline of Chivalry». Chaucer Review. Vol. 7, 1973.

MANn, J.: Chaucer and the Medieval Estates Satire. Cambridge: Cambridge at the University Press, 1973. 
Nicholson, R. H.: «Theseus's Ordinaunce»: Justice and Ceremony in The Knight's Tale. Chaucer Review. Vol II, 1987.

Olson, C. C.: «Chaucer and fourteenth Century Society». Companion to Chaucer Studies. B. Rowland (ed.) Oxford: Oxford University Press, 1968.

RoBinson, F. N. (ed.): Works of Chaucer. Oxford: Oxford University Press. Fifth impression, 1957-1987.

Russel, F. H.: The Just War in the Middle Ages. Cambridge: Cambridge University Press, 1975.

SKEAT, W. W.: The Complete Works of Geoffrey Chaucer. 7 vols. Oxford, 1894-7: repr. 1972.

Whiтtоcк, T. A.: A reading of The Canterbury Tales. Cambridge. Cambridge University Press, 1968. 